\title{
Similar periprosthetic joint infection rates after and before a national infection control program: a study of 45,438 primary total knee arthroplasties
}

\author{
Olof THOMPSON ${ }^{1}$, Annette W-DAHL ${ }^{2,3}$, Viktor LINDGREN ${ }^{4}$, Max GORDON ${ }^{5}$, Otto ROBERTSSON 2,3, \\ and Anna STEFÁNSDÓTTIR ${ }^{3,6}$
}

\begin{abstract}
${ }^{1}$ Infectious Diseases, Department of Clinical Sciences Lund, Lund University, Skåne University Hospital, Lund, Sweden; ${ }^{2}$ Swedish Knee Arthroplasty Register, Lund, Sweden; ${ }^{3}$ Department of Clinical Sciences Lund, Lund University, Division of Orthopedics; ${ }^{4}$ Department of Molecular Medicine and Surgery, Section of Orthopedics, Karolinska Institute, Stockholm, Sweden; ${ }^{5}$ Department of Clinical Sciences at Danderyd Hospital, Division of Orthopedics, Karolinska Institutet, Stockholm, Sweden; ${ }^{6}$ Department of Orthopedics, Skåne University Hospital, Lund, Sweden

Correspondence: olof.thompson@med.lu.se

Submitted 2021-01-29. Accepted 2021-08-01.
\end{abstract}

Background and purpose - Strenuous efforts to minimize postoperative infection rates have been made, including the Swedish nationwide initiative Prosthesis Related Infections Shall be Stopped (PRISS). This study calculated the incidence rate of periprosthetic joint infection (PJI) following primary total knee arthroplasty (TKA) before and after PRISS.

Patients and methods - All 45,438 primary TKAs registered in the Swedish Knee Arthroplasty Register (SKAR) during 2007-2008 and 2012-2013 were included. Matched data on antibiotic prescriptions were obtained from the Swedish Prescribed Drug Register (SPDR). All patients with $\geq 28$ days of continuous antibiotic treatment within 2 years of primary surgery had their medical charts reviewed to identify cases of PJI. The incidence rate was calculated by dividing the number of PJIs by the total time at risk during each time period and presented as percentages with 95\% confidence interval (CI).

Results - 644 PJIs were identified, equaling a 2-year incidence rate of $1.45 \%$ (CI 1.34-1.57). The incidence rate was $1.44 \%$ (CI $1.27-1.61$ ) before PRISS and $1.46 \%$ (CI 1.31-1.61) after. Diagnosis was made within 30 days of primary TKA in 52\%, and within 90 days in $73 \%$ of cases. 603 cases were reoperated on or revised. Median time from operation to diagnosis was 29 days (1-716), for both time periods. Debridement with exchange of the insert was performed in 32\% and 63\% of cases before and after PRISS, respectively.

Interpretation - We found similar incidence rates before and after the PRISS initiative without any statistically significant difference. Time to diagnosis was similar during both time periods. The project may have contributed to increased compliance with treatment protocols.
Periprosthetic joint infection (PJI) following arthroplasty of the knee is a serious and challenging complication with a reported incidence from $0.5 \%$ to $1.5 \%$ (Jämsen et al. 2009, Kurtz et al. 2010, Zhu et al. 2016, Troillet et al. 2017). As the number of arthroplasties is expected to rise in the coming years, so is the absolute number of PJIs (Wolford et al. 2018). Infection is the leading cause for revision within 2 years of primary surgery and preventive measures are highly prioritized internationally (ICM Philly 2018). Studies have also demonstrated rising trends of PJIs (Dale et al. 2012, Lindgren et al. 2014a, Lenguerrand et al. 2017, Springer et al. 2017).

Strenuous efforts to minimize infection rates have therefore been made, including the Swedish nationwide PRISS project (Prosthesis Related Infections Shall be Stopped) 2009-2012. PRISS included all units performing elective arthroplasty surgery, aiming at a reduction of postoperative infections in elective hip and knee arthroplasty surgery by $50 \%$ (Gustafson et al. 2014). Implemented measures included optimization of pre-, per-, and postoperative care, such as patient selection and optimization, basic hygiene procedures, adequate operational environment, perioperative prophylactic antibiotics, postoperative wound care, and early detection of suspected infections. However, the relative importance of different measures is under debate and the effects of optimization largely unknown.

In addition, incidence rates of PJI are hard to measure. National arthroplasty registers underestimate PJI incidence rates (Dale et al. 2012, Lindgren et al. 2014b, Zhu et al. 2016, Gundtoft et al. 2017), making them less suitable for infection surveillance. Multiple data sources need therefore to be used in order to reach better estimates. A novel approach, combining arthroplasty register data and data on antibiotic prescriptions with review of medical records, was described by Lindgren et al. (2014a) in assessing PJI following primary total 
hip arthroplasty (THA). This methodology was also used in a recent follow-up study evaluating the effect of PRISS on the PJI burden after primary THA (Wildeman 2021, personal communication). We have adapted this method to estimate incidence rates for PJI within 2 years following total knee arthroplasty (TKA) both before and after PRISS.

Wildeman et al. showed no effect of Priss on PJI-rates after THA (2021, personal communication). The primary aim of this study was to evaluate the effect of the PRISS project on the incidence rate of PJI following primary TKA, by calculating the cumulative incidence rate before and after the project. Secondary aims were to evaluate time to diagnosis, primary treatment method, and PJI registration in the Swedish Knee Arthroplasty Register (SKAR).

\section{Patients and methods}

\section{Data sources}

\section{The Swedish Knee Arthroplasty Register}

The Swedish Knee Arthroplasty Register (SKAR) was initiated by the Swedish Orthopedic Association in 1975. Data on primary arthroplasties and subsequent revisions is prospectively collected, and all private and public units performing knee arthroplasty surgery report to the register and participate voluntarily. Continuous validation shows a 97\% completeness of primary knee arthroplasties (Swedish Knee Arthroplasty Register 2009, 2014). Revision is defined as a new operation in a previously resurfaced knee in which 1 or more of the components are exchanged, removed, or added. Change of tibial insert, arthrodesis, and above-knee amputation are thereby revisions. Reoperations, that is other surgery not defined as revision, were not collected by the SKAR until 2014 but if a reoperation was reported it was registered. Examples of reoperations are soft-tissue or fracture surgeries of the knee, without addition, exchange, or removal of implant components. That is, a plain synovectomy would not count as a revision but be classified as a reoperation. Information on age, sex, and diagnosis was obtained from the SKAR.

\section{Swedish Prescribed Drug Register}

The Swedish Prescribed Drug Register (SPDR) has been active since 2005 and is, like all Swedish healthcare and quality registers, based on the unique national personal identification number, enabling data matching between registers. All pharmacies, public and private, have a mandatory reporting obligation to SPDR of all dispensed prescriptions, and data is automatically collected via direct transfer of computer records. SPDR holds information on dispensed item, ATC code, amount, dosage, dates of prescription and dispensing, instructions from prescribing physician, etc. All prescription drugs are included in SPDR, accounting for $84 \%$ of the total drug utilization in Sweden. Since no systemic antibiotics are sold over the counter in Sweden, the SPDR includes all out- patient systemic antibiotics, accounting for $90 \%$ of the total utilization (Wettermark et al. 2007).

\section{Methods}

All 45,438 cases registered in SKAR for primary TKA, regardless of diagnosis, type of implant, or fixation method, during 2007-2008 and 2012-2013 were included in the study. Each individual TKA was counted separately, including bilateral procedures. This cohort was matched with the SPDR using the personal identification number, and data regarding dispensed antibiotic prescriptions within 2 years of primary surgery was extracted. Antibiotics with ATC-codes J01, J02, J04, and P01 were included. Prolonged courses of antibiotics have long been part of the standardized treatment of PJI, and we therefore limited inclusion to patients who had antibiotics dispensed equal to at least 4 weeks of continuous treatment within 2 years of primary TKA (Table 1, see Supplementary data). Cases where a clear indication for antibiotic treatment other than PJI was stated on the prescription (e.g., pneumonia, urinary tract infection), were manually excluded. Cases with a suspected infection registered in SKAR that were not identified through the SPDR were also included in the analysis $(n=63)$.

By this approach we identified 2,505 cases. A questionnaire regarding each of the identified patients was sent to physicians at the unit performing the primary surgery (78 units), in which we asked for retrospective review of the patient records. Confirmation or exclusion of PJI was thus obtained. If a PJI diagnosis was found, additional information regarding date of diagnosis, clinical signs, surgical treatment, microbiological results, duration of antibiotic treatment, and outcome was also obtained. Where a defined date for diagnosis was missing, we used the date for first surgical intervention. We included all patients where the physician reported a PJI, recognizing the diagnostic difficulties and the variations in PJI definitions over time. In cases where no further information was present at the primary operating unit, the follow-up unit was identified and additional review of medical records at the follow-up unit was performed. 2,391 reports were returned (95\%) and 644 PJI cases identified (Figure 1).

\section{Statistics}

Descriptive statistics are presented as means (SD) or medians (ranges), and numbers (\%). The incidence rate was calculated by dividing the number of PJIs with the total time at risk during the first 2 years of each time period, following patients for a maximum of 2 years or until death, migration, or reoperation for causes other than infection. The respective 2-year cumulative incidence rate was then derived by multiplying the incidence rate by 2 years and was presented in percentage with $95 \%$ confidence interval (CI). We used Cox regression to assess the hazard ratio (HR) for PJI between the time periods and included age, sex, diagnosis (dichotomized on osteoarthritis $[(\mathrm{OA}]$ or not), and fixation of the TKA (cemented or uncemented) in the final model. 


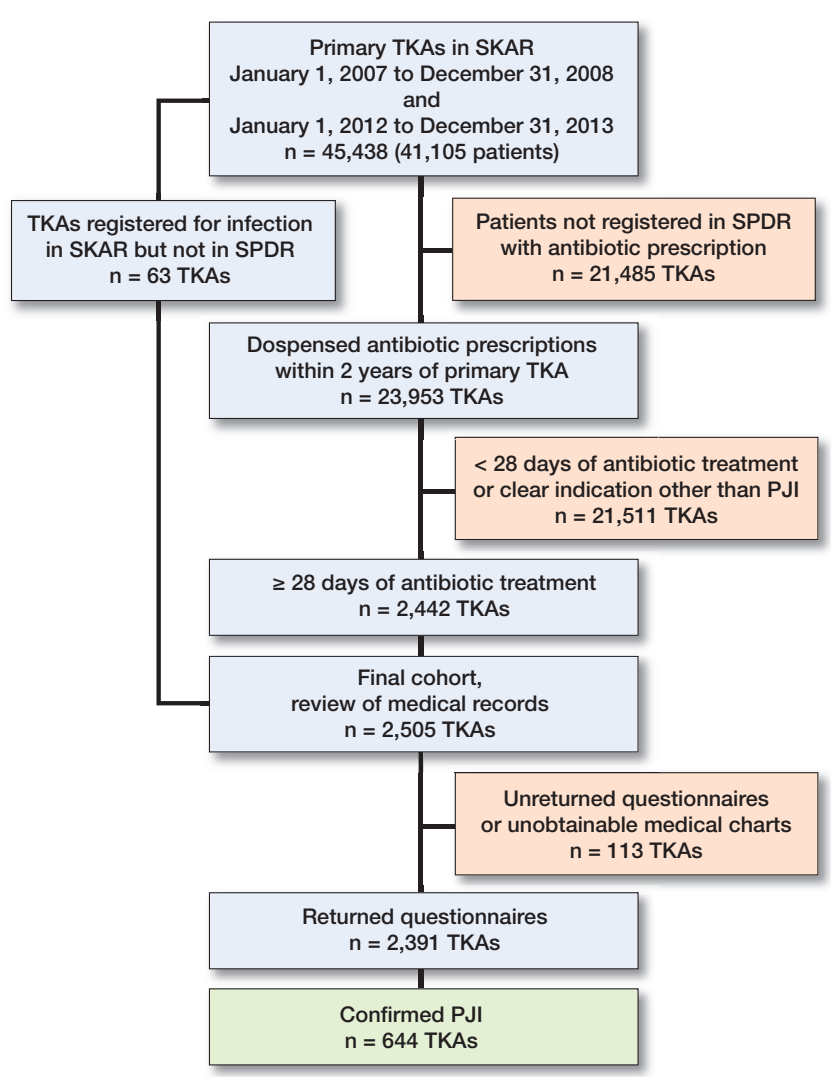

Figure 1. Study flowchart. TKA: total knee arthroplasty; SKAR: Swedish Knee Arthroplasty Register; SPDR: Swedish Prescribed Drug Register; PJI: periprosthetic joint infection.

Table 2. Characteristics of all primary total knee arthroplasty (TKA) cases during 2007-2008 and 2012-2013. Values are number (\%) unless otherwise stated

\begin{tabular}{lrrr}
\hline All TKAs & $\begin{array}{c}\text { Total } \\
\mathrm{n}=45,438\end{array}$ & $\begin{array}{c}2007-2008 \\
\mathrm{n}=19,940\end{array}$ & $\begin{array}{r}2012-2013 \\
\mathrm{n}=25,498\end{array}$ \\
\hline Male sex & $18,793(41)$ & $7,937(40)$ & $10,837(43)$ \\
Age, mean (SD) & $69(9.1)$ & $70(9.1)$ & $69(9.1)$ \\
Diagnosis & & & \\
$\quad$ OA & $43,902(97)$ & $19,425(97)$ & $24,477(96)$ \\
Inflammatory & & & \\
$\quad$ joint disease & $1,005(2.2)$ & $432(2.2)$ & $573(2.2)$ \\
$\quad \begin{array}{l}\text { Posttraumatic OA } \\
\text { Other }\end{array}$ & $215(0.5)$ & $21(0.1)$ & $194(0.8)$ \\
Fixation & $316(0.7)$ & $62(0.3)$ & $254(1.0)$ \\
$\quad$ Cemented & $43,882(97)$ & $19,676(99)$ & $24,206(95)$ \\
$\quad$ Uncemented & $1,430(3.1)$ & $189(0.9)$ & $1,241(4.9)$ \\
$\quad$ Unknown & $126(0.3)$ & $75(0.4)$ & $51(0.2)$ \\
\hline OA: Osteoarthritis. & & &
\end{tabular}

Study data was constructed and managed using REDCap (Research Electronic Data Capture), a secure web-based data capture application hosted at the Faculty of Medicine, Lund University. For statistical analysis IBM SPSS Statistics software (v.26) for macOS was used (IBM Corp, Redmond, WA, USA.
Table 3. Characteristics of all periprosthetic joint infection (PJI) cases during 2007-2008 and 2012-2013. Values are number (\%) unless otherwise stated

\begin{tabular}{|c|c|c|c|c|c|}
\hline \multirow[b]{2}{*}{ PJl cases } & \multicolumn{3}{|c|}{ Verified PJI cases } & \multicolumn{2}{|c|}{ Missing cases ${ }^{a}$} \\
\hline & $\begin{array}{c}\text { Total } \\
n=644\end{array}$ & $\begin{array}{c}2007- \\
2008 \\
n=280\end{array}$ & $\begin{array}{c}2012- \\
2013 \\
n=364\end{array}$ & $\begin{array}{c}2007- \\
2008 \\
n=74\end{array}$ & $\begin{array}{c}2012- \\
2013 \\
n=39\end{array}$ \\
\hline Male sex & $379(59)$ & $162(58)$ & $217(60)$ & 38 & 25 \\
\hline Age, mean (SD) & $69(10)$ & $70(10)$ & $69(10)$ & $72(9.4)$ & $65(12)$ \\
\hline \multicolumn{6}{|l|}{ Diagnosis } \\
\hline OA & $595(92)$ & $257(92)$ & $338(93)$ & 72 & 36 \\
\hline \multicolumn{6}{|c|}{ Inflammatory joint } \\
\hline Posttraumatic OA & $10(1.6)$ & $2(0.7)$ & $8(2.2)$ & 0 & 0 \\
\hline Other & $8(1.2)$ & $2(0.7)$ & $6(1.6)$ & 0 & 1 \\
\hline \multicolumn{6}{|l|}{ Fixation } \\
\hline Cemented & $623(97)$ & $273(98)$ & $350(96)$ & 74 & 39 \\
\hline Uncemented & $17(2.6)$ & $5(1.8)$ & 12 (3.3) & 0 & 0 \\
\hline Unknown & $4(0.6)$ & $2(0.7)$ & $2(0.5)$ & 0 & 0 \\
\hline
\end{tabular}

a Cases with unobtainable medical charts.

\section{Ethics, funding, and conflicts of interest}

Study approval was granted November 2, 2016 by the Ethics Review Board at Lund University (Dnr 2016/28). The need for informed consent was waived by the Ethics Review Board.

The study was financially supported by regional research grants from Region Skåne and by Löf, the Swedish patient insurance. The authors declare no conflicts of interest.

\section{Results}

Of the 45,438 TKAs included, $96.6 \%$ were performed due to OA and $96.6 \%$ had the components fixed with bone cement. Between the time periods 2007-2008 and 2012-2013 there was an increasing proportion of males (39.8\% vs. $42.5 \%)$, a slight decrease in age (69.6 vs. 68.9 years), and an increase of un-cemented procedures ( $0.9 \%$ vs. $4.9 \%$ ) (see Table 2 and Table 3 , which includes demographics for missing cases).

The overall 2-year incidence rate was $1.45 \%$ (CI 1.34-1.57): $1.44 \%$ (CI 1.27-1.61) for cases operated in 2007-2008 and $1.46 \%$ (CI 1.31-1.61) in 2012-2013.

Date for diagnosis was available in 638 of 644 identified PJIs. Median time from operation to diagnosis was 29 days (1-716), for both time periods. $52 \%$ of patients were diagnosed within the first 30 postoperative days, and $73 \%$ of cases were diagnosed within the first 90 days. Time to diagnosis was similar during both time periods (Figure 2).

Data on surgical treatment was available in 639 cases, of which 603 had surgery performed as part of PJI treatment. Preference for surgical intervention changed between the 2 periods, most notably an increase in the proportion of debridements with exchange of insert in 2012-2013 (32\% vs. 63\%) and a subsequent decrease in debridements without exchange of insert (33\% vs. $18 \%$ ) and arthroscopic procedures (16\% vs. 


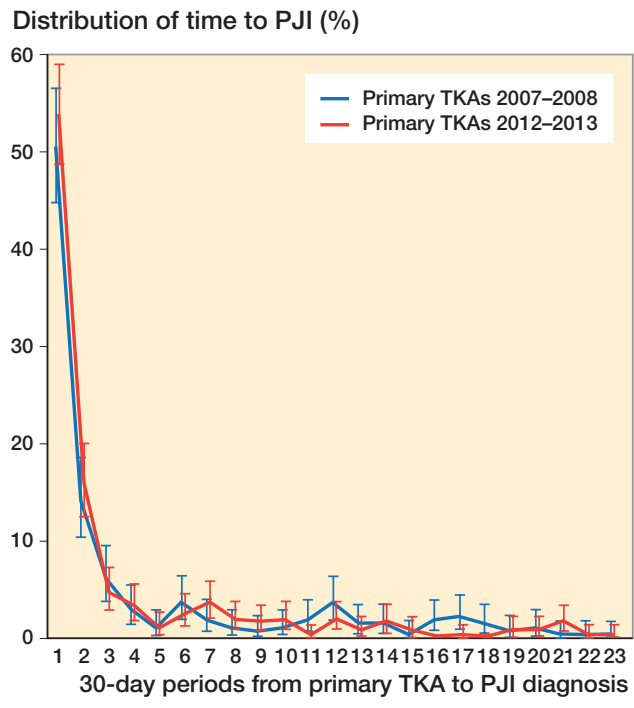

Figure 2. Time from primary TKA to diagnosis of periprosthetic joint infection (PJI) during 2007-2008 and 2012-2013. Each value represents a 30-day period.

Table 4. PJI cases, cumulative incidence rates, and first surgical treatment for PJI before and after the PRISS project

\begin{tabular}{|c|c|c|c|}
\hline PJI cases & $\begin{array}{c}\text { Total } \\
n=644\end{array}$ & $\begin{array}{c}2007-2008 \\
n=280\end{array}$ & $\begin{array}{c}2012-2013 \\
n=364\end{array}$ \\
\hline Person time, days & $32,344,751$ & $14,177,494$ & $18,167,257$ \\
\hline \multicolumn{4}{|l|}{ Cumulative 2-year } \\
\hline incidence, \% & 1.45 & 1.44 & 1.46 \\
\hline$(95 \% \mathrm{Cl})$ & $(1.34-1.57)$ & $(1.27-1.61)$ & $(1.31-1.61)$ \\
\hline \multicolumn{4}{|c|}{ Hazard ratio of PJI $(95 \% \mathrm{Cl})$} \\
\hline $\begin{array}{c}\text { non-adjusted } \\
(95 \% \mathrm{Cl})\end{array}$ & NA & 1 (ref) & $\begin{array}{c}1.01 \\
(0.86-1.17)\end{array}$ \\
\hline $\begin{array}{l}\text { adjusted }^{\text {a }} \\
(95 \% \mathrm{Cl})\end{array}$ & NA & 1 (ref) & $\begin{array}{c}0.98 \\
(0.84-115)\end{array}$ \\
\hline \multicolumn{4}{|l|}{ First surgical intervention, $\mathrm{n}(\%)$} \\
\hline \multicolumn{4}{|l|}{ Revisions } \\
\hline \multicolumn{4}{|l|}{ Debridement with } \\
\hline exchange of plastic & $315(49)$ & $88(31)$ & $227(62)$ \\
\hline One-stage exchange & $8(1.2)$ & $1(0.4)$ & $7(1.9)$ \\
\hline \multicolumn{4}{|l|}{ Two-stage exchange, } \\
\hline removal & $45(7.0)$ & $21(7.5)$ & $24(6.6)$ \\
\hline Arthrodesis & $3(0.5)$ & $3(1.1)$ & $0(0)$ \\
\hline Resection arthroplasty & $1(0.2)$ & $1(0.4)$ & $0(0)$ \\
\hline Amputation & $1(0.2)$ & $1(0.4)$ & $0(0)$ \\
\hline \multicolumn{4}{|l|}{ Reoperations } \\
\hline Arthroscopy & $73(11)$ & $45(16)$ & $28(7.7)$ \\
\hline \multicolumn{4}{|l|}{ Debridement without } \\
\hline exchange of insert & $156(24)$ & $91(33)$ & 65 (18) \\
\hline Other/unknown intervention & $2(0.3)$ & $2(0.7)$ & $0(0)$ \\
\hline No surgery & $36(5.6)$ & $24(8.6)$ & $12(3.3)$ \\
\hline Missing intervention data & $4(0.6)$ & $3(1.1)$ & $1(0.3)$ \\
\hline
\end{tabular}

$7.7 \%)$. The proportion of patients treated without surgery also decreased between the time periods ( $8.7 \%$ vs. $3.3 \%$ ) (Table 4$)$.

In the SKAR, few of the reoperations were registered as infections, although an increasing number of both reop-
Table 5. PJI capture-rate of the SKAR stratified by type of intervention. Values are number (percentage captured)

\begin{tabular}{|c|c|c|c|c|c|c|}
\hline \multirow[b]{2}{*}{ Factor } & \multicolumn{2}{|c|}{ 2007-2008 } & \multicolumn{2}{|c|}{ 2012-2013 } & \multicolumn{2}{|c|}{ Total } \\
\hline & Verified & SKAR & Verified & SKAR & Verified & SKAR \\
\hline Reoperations & 136 & $35(26)$ & 93 & $48(52)$ & 229 & $83(36)$ \\
\hline Revisions & 114 & $74(65)$ & 258 & $200(78)$ & 372 & $274(74)$ \\
\hline Total & 250 & $109(44)$ & 351 & 248 (71) & 601 & 357 (59) \\
\hline
\end{tabular}

erations and revisions were registered during the latter time period (Table 5). Overall capture rate, including all reoperated cases, was 59\% (357/601). 86 cases registered as infection in SKAR were found not to have a PJI with our study method.

\section{Discussion}

In this population-based, nationwide study we found an overall 2-year cumulative incidence rate of PJI after primary TKA of $1.45 \%$, which was similar during the 2 studied periods, 2007-2008 and 2012-2013. As infection reduction was the stated goal of the national infection-control initiative PRISS, this is disappointing.

Several factors influence the risk of PJI and can be divided into 3 categories: patient-related (as for example comorbidities, obesity, sex, and smoking), operating environment (procedures and facilities), and surgical (surgical technique, prophylactic antibiotics, and postoperative care) (Pulido et al. 2008, Kurtz et al. 2010, Adeli and Parvizi 2012, Namba et al. 2013). While some of these risk factors are relatively easy to optimize, such as correct use of prophylactic antibiotics, others are more difficult or impossible to affect (e.g., comorbidities, obesity, and patient sex). In our study male sex and non-OA indication was more common among infected cases, as expected. Furthermore, we saw an increased proportion of male patients, uncemented TKAs as well as post-traumatic OA indication during 2012-2013, possibly counteracting the effects of infection control improvements between study periods (Namba et al. 2013). However, the results remained the same between the studied periods when we adjusted for sex, age, OA, and fixation (see Table 4). SKAR began an extended registration of patient- and surgery-related factors in 2009 (including BMI, ASA score, and prophylactic antibiotics), which precludes comparison of these factors between the time periods in our study.

Increasing antibiotic resistance has also been proposed as an explanation for increasing PJI incidence (Dale et al. 2012), though actual evidence is lacking. In support of this hypothesis are previous findings of increasing proportions of coagulase negative staphylococci (CoNS) in PJIs over time (Stefánsdóttir et al. 2009) as well as increasing rates of methicillin resistance among CoNS (Lutro et al. 2014). A recent Danish study, however, found no evidence of increasing rates of beta-lactam 
resistance among PJIs from hip arthroplasties (Gundtoft et al. 2017). Hopefully more light can be shed on this question as we plan to investigate the microbiology of the PJI population in future work.

Heightened awareness among clinicians regarding PJI is another factor suggested to increase cumulative revision rates in register reports (Dale et al. 2012, Swedish Knee Arthroplasty Register 2014, Springer et al. 2017). We believe, however, that the method we used did not include the exchange of insert revisions that resulted in a non-PJI diagnosis, thereby capturing "true" PJIs rather than just increased diagnostic activity among clinicians. It is, however, possible that more "false positive" diagnoses are being made partly because more diagnostic procedures are performed. Given the severe implications of a missed PJI diagnosis, it is also possible that clinicians choose to over-diagnose rather than under-diagnose, when faced with inconclusive cases. Further, the PRISS project may have led to increased PJI vigilance among clinicians, contributing to the increased proportion of debridement and irrigation with prosthetic retention (DAIR) procedures in the latter time period.

The main limitation of this investigation is the use of outpatient antibiotic prescription data to identify potential PJI patients. Though data loss in SPDR is presumably zero, some patients may have been missed due to death before discharge or complete in-hospital treatment, as well as patients not collecting antibiotic prescriptions for various reasons. The number is not known but we believe these patients to be few. We have no reason to assume that major changes have occurred in this respect between the time periods in the study but acknowledge that complete in-hospital treatment may have been more common before PRISS. Though register data was collected prospectively, the medical records were reviewed retrospectively, which presents another limitation. Furthermore, the diagnosis of PJI is a clinical challenge, being based on a combination of clinical findings, cultures, and laboratory tests. In recent years international consensus on PJI definitions and diagnosis has been developed but was not present during the first time period in our study. Clinical practice and diagnostic work-up has therefore changed over the years, possibly affecting the results in our study. Medical records were reviewed locally by many different physicians. Lack of coherence in reporting between reviewers is therefore a possibility and constitutes another limitation.

In 113 cases medical records were unobtainable or questionnaires not returned. It is possible that some cases of PJI have been missed in this group, resulting in a slight under-estimation of the cumulative incidence. When we adopted a bestor worst-case scenario (counting none or all missing cases as PJIs), the result remained, with small difference in incidence between the studied periods.

Several other studies estimating the incidence of PJI after TKA exist, reaching different conclusions (Katz et al. 2004, Pulido et al. 2008, Jämsen et al. 2009, Kurtz et al. 2010, Zhu et al. 2016, Lenguerrand et al. 2017, Troillet et al. 2017, Baier et al. 2019). Comparison is impeded, however, by disparities in setting, definitions, patient sampling, and follow-up time. Our estimate of $1.45 \%$ 2-year incidence is slightly higher than other studies with similar sample sizes calculating rates between $0.32 \%$ and $1.16 \%$ (Jämsen et al. 2009, Kurtz et al. 2010, Lenguerrand et al. 2017). These studies, although large, were all based solely on administrative datasets, and are therefore at risk of low sensitivity and, hence, underestimation. Studies derived from single centers or specialized institutions have based their estimates on review of patient records, which increases the precision (Pulido et al. 2008, Jämsen et al. 2010, Zhu et al. 2016, Baier et al. 2019), but the results are difficult to generalize to a broader population. Our study has the advantage of being based on a nationwide cohort, where the treatment of the infection was used to identify patients, and retrospective review of the medical records determined whether the PJI diagnosis was present. We believe all these factors to increase the credibility of our assessment.

Our finding of similar incidence rates before and after PRISS is supported in the recent study by Wildeman et al. (2021), which found no effect of PRISS on the incidence of PJI following primary THA. Further, the time to diagnosis was similar before and after PRISS, concurrent with our results.

$44 \%$ of all PJIs in this study were not captured by the registration in the SKAR, which thereby underestimated PJI incidence. This is well in line with several other studies (Jämsen et al. 2009, Lindgren et al. 2014b, Gundtoft et al. 2015, Zhu et al. 2016). However, the SKAR was not designed to find all cases of infection but is focused on revisions (defined as exchange, addition, or removal of any part of the prosthesis), which exhibited a capture rate of infected revisions of $73 \%$. Further, 8.7\% of PJIs during 2007-2008 were not operated as part of PJI treatment, and were, subsequently, not possible for the SKAR to identify. Given the relative difficulty of making a PJI diagnosis and the focus of arthroplasty registers on revision rates, we believe these registers to be inherently prone to underestimate PJI rates. 86 cases that were registered as infections in the SKAR were not reported as PJIs in our study. 28 of these cases were infected after revision for other causes, while 20 were reported as superficial infections $(n=20)$. In 38 cases no infection was reported. These discrepancies may in part reflect the way infections are defined in the SKAR, where revisions for suspected infections and revisions following wound rupture are registered as infections. Changes in treatment practice also make it difficult to use pseudo-variables such as revision rates to monitor PJI incidence rates. This was illustrated in our study, where a substantial shift in surgical treatment practice occurred between the 2 time periods. An increased proportion of debridement with exchange of modular parts was found during 2012-2013, while arthroscopic procedures and debridement without exchange of modular parts decreased. This also resulted in improved capture rates of infections 
in the SKAR. Since the proportion of early $(<30$ days of implantation) PJIs was almost unchanged between study periods, we believe these changes in surgical treatment to reflect changes in clinical practice, rather than changes in PJI epidemiology. The change in clinical practice also coincided with the development of national and international treatment guidelines on PJI, where use of thorough debridement with exchange of modular parts was (and still is) recommended as first-line treatment for most early postoperative PJIs (ICM Philly 2018).

The method used in this study was previously proposed as a method for infection surveillance (Lindgren et al. 2014a) but, in our experience, the process is too labor intensive to be a truly viable option for surveillance purposes. The use of multiple data sources combined with machine learning is likely to become a future alternative but, until reliable methods are in place, we suggest that efforts should be made to increase the capture rate in the registers.

\section{Conclusion}

Despite an ambitious infection control project, we found similar incidence rates of PJI within 2 years after TKA before and after the PRISS initiative. The reasons for this are unclear, but changes in patient demographic factors or bacterial resistance patterns may contribute. Time to diagnosis was similar during both time periods. The project may have contributed to the increased compliance with treatment protocols seen after PRISS. Incidence rates were underestimated in the SKAR, as expected.

AS, AWD, and OR conceived the study. VL and MG developed the methodology, and MG wrote the data extraction script for SPDR. OR extracted data from SKAR. OT collected questionnaire data, performed data analysis, and wrote the manuscript.

The authors would like to thank the SKAR contact physicians who contributed to this study by returning patient questionnaires.

Acta thanks Ove Furnes and Tina Strømdal Wik for help with peer review of this study.

Adeli B, Parvizi J. Strategies for the prevention of periprosthetic joint infection. J Bone Joint Surg Br 2012; 94 (11 Suppl A): 42-6.

Baier C, Adelmund S, Schwab F, Lassahn C, Chaberny I F, Gossé F, Vonberg R P, Ebadi E. Incidence and risk factors of surgical site infection after total knee arthroplasty: results of a retrospective cohort study. Am J Infect Control 2019; 47(10): 1270-2.

Dale H, Fenstad A M, Hallan G, Havelin L I, Furnes O, Overgaard S, Pedersen A B, Kärrholm J, Garellick G, Pulkkinen P, Eskelinen A, Mäkelä K, Engesæter L B. Increasing risk of prosthetic joint infection after total hip arthroplasty. Acta Orthop 2012; 83(5): 449-58.
Gundtoft P H, Overgaard S, Schønheyder H C, Møller J K, KjærsgaardAndersen P, Pedersen A B. The "true" incidence of surgically treated deep prosthetic joint infection after 32,896 primary total hip arthroplasties. Acta Orthop 2015; 86(3): 326-34.

Gundtoft P H, Pedersen A B, Schønheyder H C, Møller J K, Overgaard S. One-year incidence of prosthetic joint infection in total hip arthroplasty: a cohort study with linkage of the Danish Hip Arthroplasty Register and Danish Microbiology Databases. Osteoarthr Cartil 2017; 25(5): 685-93.

Gustafson P, Schultz T, Stefánsdóttir A. PRISS - Prosthesis-Related Infections Shall be Stopped - a national, interdisciplinary collaboration for safer prosthetic knee and hip operations 2014. Available at: https://lof.se/filer/ PRISS-final-report-oct2014.pdf

ICM Philly 2nd International Consensus Meeting (ICM) on Musculoskeletal Infection 2018. Hip \& Knee 2018 [Online]. Available from: https:// icmphilly.com/hip-knee/ (Accessed January 1, 2021).

Jämsen E, Huotari K, Huhtala H, Nevalainen J, Konttinen Y T. Low rate of infected knee replacements in a nationwide series: is it an underestimate? Review of the Finnish Arthroplasty Register on 38,676 operations performed in 1997 through 2003. Acta Orthop 2009; 80(2): 205-12.

Jämsen E, Varonen M, Huhtala H, Lehto M U K, Lumio J, Konttinen Y T, Moilanen T. Incidence of prosthetic joint infections after primary knee arthroplasty. J Arthroplasty 2010; 25(1): 87-92.

Katz J N, Barrett J, Mahomed N N, Baron J A, Wright R J, Losina E. Association between hospital and surgeon procedure volume and the outcomes of total knee replacement. J Bone Joint Surg Am 2004; 86(9): 1909-16.

Kurtz S M, Ong K L, Lau E, Bozic K J, Berry D, Parvizi J. Prosthetic joint infection risk after TKA in the Medicare population. Clin Orthop Relat Res 2010; 468(1): 52-6.

Lenguerrand E, Whitehouse M R, Beswick A D, Toms A D, Porter M L, Blom A W. Description of the rates, trends and surgical burden associated with revision for prosthetic joint infection following primary and revision knee replacements in England and Wales: an analysis of the National Joint Registry for England, Wales, Northern Ire. BMJ Open 2017; 7(7): e014056.

Lindgren V, Gordon M, Wretenberg P, Kärrholm J, Garellick G. Deep infection after total hip replacement: a method for national incidence surveillance. Infect Control Hosp Epidemiol 2014a; 35(12): 1491-6.

Lindgren V, Gordon M, Wretenberg P, Kärrholm J, Garellick G. Validation of reoperations due to infection in the Swedish Hip Arthroplasty Register. BMC Musculoskelet Disord 2014b; 15(1): 384.

Lutro O, Langvatn H, Dale H, Schrama J C, Hallan G, Espehaug B, Sjursen H, Engesæeter L B. Increasing resistance of coagulase-negative staphylococci in total hip arthroplasty infections: 278 THA-revisions due to infection reported to the Norwegian Arthroplasty Register from 1993 to 2007. Adv Orthop 2014; 2014: 580359.

Namba R S, Inacio M C S, Paxton E W. Risk factors associated with deep surgical site infections after primary total knee arthroplasty: an analysis of 56,216 knees. J Bone Joint Surg 2013; 95(9): 775-82.

Pulido L, Ghanem E, Joshi A, Purtill J J, Parvizi J. Periprosthetic joint infection: the incidence, timing, and predisposing factors. Clin Orthop Relat Res 2008; 466(7): 1710-5.

Springer B D, Cahue S, Etkin C D, Lewallen D G, McGrory B J. Infection burden in total hip and knee arthroplasties: an international registry-based perspective. Arthroplast Today 2017; 3(2): 137-40.

Stefánsdóttir A, Johansson D, Knutson K, Lidgren L, Robertsson O. Microbiology of the infected knee arthroplasty: report from the Swedish Knee Arthroplasty Register on 426 surgically revised cases. Scand J Infect Dis 2009; 41(11-12): 831-40.

Swedish Knee Arthroplasty Register. Annual report 2009. Available at https://www.myknee.se/en/publications/annual-reports.

Swedish Knee Arthroplasty Register. Annual report 2014. Available at https://www.myknee.se/en/publications/annual-reports.

Troillet N, Aghayev E, Eisenring M C, Widmer A F. First Results of the Swiss National Surgical Site Infection Surveillance Program: who seeks shall find. Infect Control Hosp Epidemiol 2017; 38(6): 697-704. 
Wettermark B, Hammar N, MichaelFored C, Leimanis A, Otterblad Olausson P, Bergman U, Persson I, Sundström A, Westerholm B, Rosén M. The new Swedish Prescribed Drug Register: opportunities for pharmacoepidemiological research and experience from the first six months. Pharmacoepidemiol Drug Saf 2007; 16(7): 726-35.

Wolford H M, Hatfield K M, Paul P, Yi S H, Slayton R B. The projected burden of complex surgical site infections following hip and knee arthroplasties in adults in the United States, 2020 through 2030. Infect Control Hosp Epidemiol 2018; 39(10): 1189-95.

Zhu M, Ravi S, Frampton C, Luey C, Young S. New Zealand Joint Registry data underestimates the rate of prosthetic joint infection. Acta Orthop 2016; 87(4): 346-50. 


\section{Supplementary data}

Table 1. List of antibiotics and doses needed to calculate 28 days of treatment

\begin{tabular}{|c|c|c|c|}
\hline Antibiotic & ATC code & Daily dose & $\begin{array}{l}28 \text { days of } \\
\text { treatment }\end{array}$ \\
\hline Amoxicillin & J01CA04 & $750 \mathrm{mg} 1 \times 3$ & 84 \\
\hline \multicolumn{4}{|l|}{ Amoxicillin and } \\
\hline clavulanic acid & J01CR02 & $850 / 125 \mathrm{mg} 1 \times 3$ & 84 \\
\hline Azithromycin & J01FA10 & $250 \mathrm{mg} 1 \times 1$ & 28 \\
\hline Cefadroxil & J01DB05 & $1 \mathrm{~g} 1 \times 2$ & 56 \\
\hline Ceftaroline fosamil & J01DI02 & $600 \mathrm{mg} 1 \times 2$ & 56 \\
\hline Ceftibuten & J01DD14 & $400 \mathrm{mg} 1 \times 1$ & 28 \\
\hline Ceftriaxone & J01DD04 & $2 \mathrm{~g} 1 \times 1$ & 28 \\
\hline Cefuroximaxetil & J01DC02 & $250 \mathrm{mg} 1 \times 2$ & 56 \\
\hline Ciprofloxacin & J01MA02 & $250 \mathrm{mg} 1 \times 2$ & 56 \\
\hline Clarithromycin & J01FA09 & $250 \mathrm{mg} 1 \times 2$ & 56 \\
\hline Clindamycin & J01FF01 & $300 \mathrm{mg} 1 \times 3$ & 84 \\
\hline Daptomycin & J01XX09 & $350 \mathrm{mg} 1 \times 1$ & 28 \\
\hline Doxycycline & J01AA02 & $100 \mathrm{mg} 2 \times 1$ & 56 \\
\hline Ertapenem & J01DH03 & $1 \mathrm{~g} 1 \times 1$ & 28 \\
\hline Erythromycin & J01FA01 & $250 \mathrm{mg} 2 \times 2$ & 112 \\
\hline Fluconazole & J02AC01 & $100 \mathrm{mg} 2 \times 1$ & 56 \\
\hline Flucloxacillin & J01CF05 & $500 \mathrm{mg} 2 \times 3$ & 84 \\
\hline Fusidic acid & J01XC01 & $250 \mathrm{mg} 2 \times 3$ & 168 \\
\hline Levofloxacin & J01MA12 & $500 \mathrm{mg} 1 \times 1$ & 28 \\
\hline Linezolid & J01XX08 & $600 \mathrm{mg} 1 \times 2$ & 56 \\
\hline Meropenem & J01DH02 & $500 \mathrm{mg} 1 \times 2$ & 56 \\
\hline Metronidazole & P01AB01 & $400 \mathrm{mg} 1 \times 3$ & 84 \\
\hline Moxifloxacin & J01MA14 & $400 \mathrm{mg} 1 \times 1$ & 28 \\
\hline Norfloxacin & J01MA06 & $400 \mathrm{mg} 1 \times 2$ & 56 \\
\hline Phenoxymethylpenicillin & J01CE02 & $1 \mathrm{~g} 2 \times 3$ & 168 \\
\hline Rifampicin & J04AB02 & $600 \mathrm{mg} 1 \times 1$ & 28 \\
\hline Roxithromycin & J01FA06 & $150 \mathrm{mg} 1 \times 2$ & 56 \\
\hline \multicolumn{4}{|l|}{ Sulfamethoxazole } \\
\hline and trimethoprim & J01EE01 & $160 / 800 \mathrm{mg} 1 \times 2$ & 56 \\
\hline Teicoplanin & J01XA02 & $400 \mathrm{mg} 1 \times 1$ & 28 \\
\hline Telithromycin & J01FA15 & $400 \mathrm{mg} 1 \times 2$ & 56 \\
\hline Vancomycin & J01XA01 & $1 \mathrm{~g} 1 \times 2$ & 56 \\
\hline
\end{tabular}

